CDD: 115

\title{
THE MATHEMATICS OF MCTAGGART'S PARADOX
}

\author{
DOMENICO MANCUSO
}

Università di Urbino (ITALY)

Université de Paris IV - Sorbonne (FRANCE)

albatros91@yahoo.com

Received: 14.09.2011 ; Accepted: 19.08.2012

\begin{abstract}
Mc Taggart's celebrated proof of the unreality of time is a chain of implications whose final step asserts that the A-series (i.e. the classification of events as past, present or future) is intrinsically contradictory. This is widely believed to be the heart of the argument, and it is where most attempted refutations have been addressed; yet, it is also the only part of the proof which may be generalised to other contexts, since none of the notions involved in it is specifically temporal. In fact, as I show in the first part of the paper, McTaggart's refutation of the A-series can be easily interpreted in mathematical terms; subsequently, in order to strengthen my claim, I apply the same framework by analogy to the cases of space, modality, and personal identity. Therefore, either McTaggart's proof as a whole may be extended to each of these notions, or it must embed some distinctly temporal element in one of the steps leading up to the contradiction of the A-series. I conclude by suggesting where this element might lay, and by hinting at what I believe to be the true logical fallacy of the proof.
\end{abstract}

Keywords: McTaggart's paradox. A-series. Infinite regress. Modality. Change.

In the last fifty years, McTaggart's proof of the unreality of time and particularly the passage on the contradiction of the A-series - has been repeatedly examined under many different perspectives. Yet, refutations and defences of his argument have rested either on phenomenological remarks, or on analyses of a rather linguistic than logical nature; what has been missing, I believe, is an adequate formalisation of the essentially mathematical structure of the mechanism governing the regress of temporal attributions.

My primary aim in this work will be to bring out such a structure, first by describing it algebraically in the form of an algorithm, and 
subsequently through a geometric representation. In the second part of the paper, I will explore some possible applications of these interpretations, most of which will concern the development of parallel paradoxes in non-temporal contexts; finally, I will examine the possible implications of my mathematical model on the general philosophical debate about the proof.

It is worth mentioning that a comprehensive assessment of Mc'Taggart's philosophy of time is not part of the purpose of this study (incidentally, this could apply to most of the philosophical literature on the paradox named after him). My work will be essentially formal, focusing on the logical framework underlying the argument for the Aseries contradiction; moreover, even as I will attempt to use such analysis as a starting point for some substantial remarks on McTaggart's paradox as a whole, I will do so within a perspective that clearly diverges from his own metaphysical presuppositions.

\section{THE CONTRADICTION OF THE A-SERIES}

Let us briefly recall the structure of Mc'Taggart's proof. The thesis that time is unreal arises from a three-step chain of implications: time entails change; change can only be made sense of in an A-series framework; and finally, the A-series is intrinsically contradictory (and so is time, consequently).

The key concept of the argument is clearly that of an A-series along with its counterpart, the B-series: to summarise, the latter could be described as an ordering of the events via the simple relationship of earlier versus later, without a privileged point of reference; the A-series, on the other hand, is the B-series equipped with a singular point moving unceasingly forward, which may be identified with the actual present.

In order to assess the soundness of the proof, both the necessity and the inconsistency of the A-series need to be discussed. Typically, McTaggart's reviewers have accepted one of these aspects while Manuscrito - Rev. Int. Fil., Campinas, v. 35, n.2, p. 233-267, jul.-dez. 2012. 
denying the other; most of the debate, however, has concentrated on the existence of the contradiction. My analysis will deal essentially with this part of the argument (which I agree with) and its possible extension to different conceptual frameworks, without attempting to give an overall evaluation of McTaggart's proof; in the end of the paper, though, I will hint at some general lines for a possible discussion of the other steps of the paradox.

The basic reason for embarking on an analysis of the A-series is that the tensed attributions it supports are essentially subjective: the actual present, my present, is always taken as an implicit point of reference. Apparently, then, the meaning of temporal predicates seems to depend on something which has in turn a temporal position, thus setting up a vicious circle. In order to avoid this, we need to establish an objective content of the A-series attributions, i.e. one that will hold independently of where the speaker "stands".

For symmetry reasons, both Mc'Taggart and his commentators have constantly chosen to start their analyses with the present predicate: I will follow their example, although the argument may be easily adjusted to the case of past or future.

Let us consider a present event E: prima facie, it may be (trivially) associated with a single tense. In some sense, though, the same event possesses in turn all three temporal attributions: it has been future, it is now present, and it will soon recede further and further into the past. Since these characteristics are mutually incompatible, we are faced with an obvious contradiction.

At first glance, there is an easy way out of the deadlock: all we need is to specify that the conflicting predicates do not apply simultaneously, but consecutively. Unfortunately, such an explanation is framed in B-series terms, and is therefore unacceptable according to the premises of Mc'Taggart's argument.

Manuscrito - Rev. Int. Fil., Campinas, v. 35, n. 2, p. 233-267, jul.-dez. 2012. 
Necessarily, then, the succession of temporal properties has to be spelt out with A-series vocabulary: $\mathrm{E}$ is future at a past moment, present at a present moment, past at a future moment.

It is plain that such a solution brings about another contradiction of the same kind as the original one, since each of the three moments I mentioned is in turn past, present and future; analysing the new predicates would then introduce a third level of contradiction, and so on, ad infinitum.

The successive steps of this infinite regress may be visualized as a sequence of propositions in the following scheme ${ }^{1}$ :

1a. Event $E$ is present.

1b. Event $E$ is present, past and future.

2a. Event $E$ is present at a present moment, past at a future moment and future at a past moment.

2b. Each of these moments is present, past and future.

3a. Each of these moments is present at a higher-level present moment m1, past at a higher-level future moment $m_{2}$ and future at a higher-level past moment $m_{3}$.

36. Each of these higher-level moments is present, past and future.

1 The source is Smith (1986), with a few significant changes. Most importantly, I have switched indexes $a$ and $b$ in the original sequence (so that, for example, Smith's proposition $2 a$ becomes $2 b, 2 b$ becomes $3 a$, etc.), in order to have decompositions of the same level correspond to nested predicates of the same length.

Furthermore, I have modified proposition $3 a$ (Smith's 3b), which was incorrect since it analysed all three attributions as "present at a present moment, past at a future moment and future at a past moment", whereas the future and the past must have different analyses. For example, the future is past at a future moment, present at another future moment and future at moments displaying in turn each of the three attributions (see picture 1).

Manuscrito - Rev. Int. Fil., Campinas, v. 35, n.2, p. 233-267, jul.-dez. 2012. 


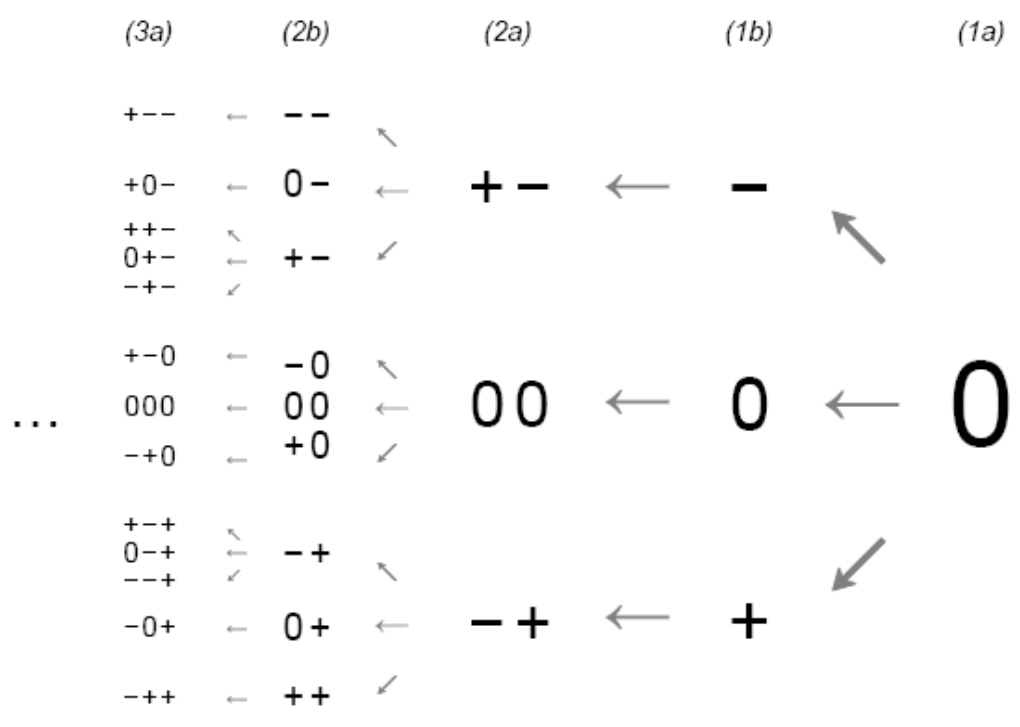

\section{$\rightarrow$ The summation rule}

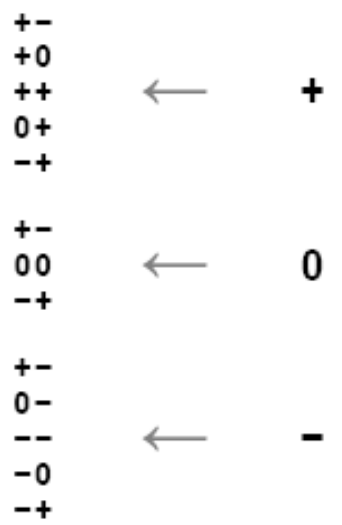

PICTURE 1. Symbolic representation of the early stages of the A-series regress. Any attribution must be read as relative to the value on its left: for example, -+ means 'future with respect to the past'.

Manuscrito - Rev. Int. Fil., Campinas, v. 35, n. 2, p. 233-267, jul.-dez. 2012. 


$$
\begin{aligned}
& \text { (3a) } \\
& \text { (2b) } \\
& +--\quad(+)-- \\
& +0- \\
& \text { (+) } 0- \\
& +-\quad(+)- \\
& \begin{array}{l}
++- \\
0+-
\end{array} \\
& (+)+- \\
& \text { (0) }+- \\
& (-)+- \\
& +-0 \quad(+)-0 \\
& \ldots 000 \longleftarrow(0) 00 \longleftarrow 00 \longleftarrow(0) 0 \\
& -+0 \quad(-)+0 \\
& \begin{array}{ll}
+-+ & (+)-+ \\
0-+ & (0)-+
\end{array} \\
& \begin{array}{ll}
0-+ & (0)-+ \\
--+ & (-)-+
\end{array} \\
& -0+\quad-10+\quad(-)+ \\
& -++\quad(-)++
\end{aligned}
$$

PiCTURE 2. Complete and incomplete analysis. The bracketed values in the $b$ columns are the implicit points of reference of the entire predicate sequence: it is easy to check that they exhaust all points on the timeline. Conversely, the $a$-columns make up an incomplete analysis of the original predicate, since all sequences relate to the actual present. The highlighted combinations exemplify the full breakdown of an attribution.

For the sake of analysis, it is preferable to replace the linguistic description of higher-level attributions with a sequence of conventional symbols. The consolidated routine among English-speaking authors is to use the initials $\mathrm{P}, \mathrm{N}, \mathrm{F}$, respectively for past, present (now) and future. I have chosen to adopt mathematical symbols instead $(-, 0$ and + ) both 
because they do not depend on any particular language and because they facilitate the algebraic interpretation I am about to give.

A further symbol is needed to represent the non-symmetric relation between any two consecutive sequences in the regress: for ease of reading, I will use an arrow. With these elements, it is now possible to construct a complete graphic representation of the early stages of the process; the result can be seen in picture 1 .

\section{The algebraic rule ${ }^{2}$}

Although descriptions of McTaggart's regress (or rather, of its initial steps) have always been carried out in an intuitive fashion, it is not difficult to produce a mechanical rule which could be iterated at any level, thus turning the whole process into a simple algorithm.

The key remark is that every predicate may be associated to a real number - or rather, more precisely, to the sign of a set of real numbers, each expressing the oriented distance between a couple of instants. Let us now consider any passage of the regress, for example the one originating from the sequence $-\mathbf{0 + + 0 + :}$ the first term on the left, which represents an unknown negative quantity, may be broken down in infinitely many ways as the sum of two reals. If we observe the signs of the addends in all such decompositions, we will see that the admissible combinations are $+\mathbf{-}, \mathbf{0 -}, \mathbf{- - ,}-\mathbf{0}$ and -+ (irrespective of the value of the initial number). As shown in picture 2 , the signs $\mathbf{0}$ and + may be similarly associated to five different couples.

Given such a split-up rule, any sequence of predicates automatically generates a set of higher-level sequences, simply by replacing the first term with the corresponding couples of attributions: in the example above, the outcome of the process will be the set $(+-$ $0++0+, 0-0++0+,--0++0+,-00++0+,-+0++0+)$.

2 This section of the paper is an extension and a development of Mancuso (2004), pp. 5-8.

Manuscrito - Rev. Int. Fil., Campinas, v. 35, n. 2, p. 233-267, jul.-dez. 2012. 
So much for the formal explanation. Yet, the main problem about McTaggart's paradox does not concern the technical rule for the regress, but rather the motive behind it: what is it that forces the left predicate to split at every stage? Why can't we just accept a given set of finite sequences as the ultimate result of the analysis?

I believe the algebraic interpretation provides a tool for addressing this kind of questions: all we need to do is specify which pair of moments must be associated to each predicate in a given sequence.

For the initial attribution, the answer is immediate: it depends on the (oriented) distance between event $\mathrm{E}$ and the actual present, and hence we may call it a subjective attribution. In the higher-order sequences, though, any term must be read with respect to the position described by the subsequence on its left - except for the first predicate, which preserves the original dependence from the moving now; since the whole chain is grounded precisely on this predicate, our sequence turns out to be still a subjective temporal attribution.

Therefore, regardless of the level of predication, an objective analysis of the initial term is systematically ruled out. On the other hand, it is possible in principle to produce what might be called a complete subjective (or quasi-objective) analysis: if we evaluate the first term in a chain of predicates with respect to all possible points of reference (which is exactly what the algebraic rule allows us to do), the result will be a string of sequences such as those highlighted in picture 2 . Considering the union of these sequences, the implicit term on the left will span over all of $\mathbf{R}$, and hence can be ignored; the remaining set of propositions is what we might take (broadly speaking) as the "objective" content of the initial sequence.

Let us now generalise to the whole of McTaggart's regress. Since the diagram branches off a single predicate, a complete $n^{\text {th }}$-order decomposition will be precisely the entire column of sequences with $n$ explicit terms (in pictures 1 and 2, the columns indexed with b). Can we say that such an analysis is also consistent? 
The definition of consistency for sets of sequences is only apparently obvious: at the first level of predication, it simply means attributions must be isolated, since they are incompatible ex bypothesi. Turning to higher orders, we may notice that some finite sequences are reducible to single attributions: namely, those containing some positive terms but no negative signs collapse onto + (and likewise for the minus sign), whereas chains of zeros are trivially equivalent to a single $\mathbf{0}$. It is clear, then, that decompositions reducing to different signs may not appear in the same column.

Apart from such very special cases, a thorough discussion of the problem would be particularly intricate, due essentially to the circular nature of the analysis - we evaluate the initial attributions in terms of each other, without introducing any non-temporal element. At any rate, however we choose to define consistency, the least we can ask for is that the sequences in a column do not exhaust all possible combinations of predicates of that particular length; otherwise, the same temporal location (e.g., present) could be trivially attached to any conceivable event.

Unfortunately, this is precisely what happens at any stage of McTaggart's regress: as we can see in picture 2 for the first and the second level, sequences in the same $b$-column (neglecting the brackets) exhibit the whole set of possible combinations of,+ 0 and - of the relevant length.

It can be shown by induction that this is true at any stage of the regress. Let us suppose the property to hold for a given $n$ : that is, the $n^{\text {th }}$-order column of index $b$ includes at least one occurrence of every sequence of $n$ terms. Each of these combinations will be associated with an implicit predicate on the left (if not more, in case of multiple occurrences); any such predicate generates a set of admissible couples, following a fixed pattern given by the summation rule (see figure 1). If we consider the second terms in every set of couples, it is easy to check that we always retrieve all three attributions: this means that each of our 
initial sequences of length $n$ will be completed on the left in three different ways at the higher level - consequently, the next $b$-column will contain all possible sequences of length $(n+1)$, quod erat demonstrandum.

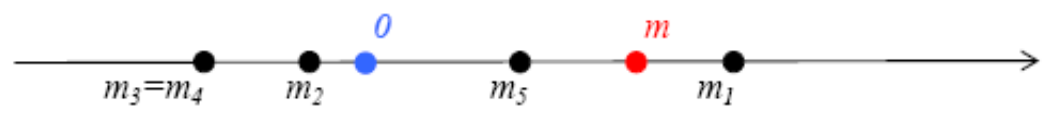

Picture 3. The geometric interpretation. The sequence of moments on the Cartesian line has to be read following the logical order $\left(0, m_{5}, m_{4}, m_{3}, m_{2}, m_{1}, m\right)$; the corresponding predicate chain is $+-=++-$.

To sum up the discussion on coherency: decompositions indexed with $b$ are complete (as they take into account all subjective points of reference), but they are not consistent; the converse holds for $a$ decompositions. The fact that the two properties may never hold together at the same stage is the motive that pushes forward the analysis of the temporal attributions ${ }^{3}$.

\section{The geometric interpretation}

An alternative way of presenting the regress of the A-series is by substituting the sequences of predicates with sequences of moments. As I mentioned, a predicate denotes the sign of the difference between the temporal coordinates of two instants - respectively corresponding to an event and a conventional point of reference ${ }^{4}$. Moreover, let us suppose each predicate in a sequence to represent a specific quantity (rather

${ }^{3}$ Besides the conflicting requirements of completeness and consistency, further background conditions might be identified in McTaggart's argument: for example, Fine (2005, pp. 271 ff.) suggests a very general version of the proof that rests on four distinct assumptions (I wish to thank an anonymous referee for pointing out this reading to me, along with Reichenbach's).

${ }^{4}$ In McTaggart's regress, the point of reference of a given sequence is in turn treated as an event in the higher-order decomposition.

Manuscrito - Rev. Int. Fil., Campinas, v. 35, n.2, p. 233-267, jul.-dez. 2012. 
than the whole set of positive or negative real numbers), and let us consider the actual present (whose coordinate is zero) as the implicit point of reference of all sequences.

We are now in a position to define a bijective correspondence between finite chains of predicates and finite chains of moments: for any element of the first set, the corresponding sequence of moments will be simply the associated series.

The connexion between the two types of successions can be easily illustrated through a geometric scheme. In the example of picture 3, a hypothetical event is located at point $m$; its position is evaluated with respect to moment $m_{1}$, which in turn has $m_{2}$ as a point of reference, and so on. Therefore, the logical (not chronological) ordering of the moments in the diagram is $0, m_{5}, m_{4}, m_{3}, m_{2}, m_{1}, m$, with the actual present as a starting point ${ }^{5}$; the corresponding predicate chain will be $+-=++-$.

Suppose now that we want to extend the latter by splitting the first term according to the summation rule: in terms of moments, this will amount to inserting a further element $m_{6}$ between 0 and $m_{5}$; iterating the procedure, an infinite sequence $\left\{m_{n}\right\}$ of real numbers is generated.

The two parallel strings of instants and predicates admit of a single unifying interpretation: the proliferation of levels can be seen as an attempt to escape the subjective attribution, which involves the actual present 0 , by interposing more and more intermediate moments between 0 and $m$; in other words, the purpose is to replace the absolute Cartesian coordinate of event $\mathrm{E}$ with an increasing set of relative

${ }^{5}$ Reichenbach (1947, \51, pp. 287-298) provided a logical analysis of compound tenses that included (cf. pp. 288-90) a representation of possible orderings of instants on the real line, limited to three levels and without a splitup rule for further extensions: in his terminology, point 0 is the point of the event, $m_{1}$ is the point of reference and $m_{2}$ is the point of speech. Prior (1967, pp. 12-13) observed that the scheme may be generalised by adding further points of reference, the first of which conicides with the point of speech.

Manuscrito - Rev. Int. Fil., Campinas, v. 35, n. 2, p. 233-267, jul.-dez. 2012. 
coordinates. Needless to say, this effort to "bridge the gap" with the actual present may never reach its target.

Incidentally, the geometric reading of the regress shows that there is nothing distinctly temporal about the contradiction of the A-series: the central issue being about the existence of an absolute frame of reference, the problem could be regarded more appropriately as a spatial one. In the final section we will explore some implications of this remark.

Another interesting corollary of the Cartesian representation has to do with the notion of "higher-level" moment. Such an expression, which is common in the literature on McTaggart's proof, might somehow suggest that each level of predication depends on the previous one - and hence, perhaps, is redundant. The full potential of this idea has been exploited by Quentin Smith (1986), who questions the paradoxical character of the A-series: after allegedly proving that McTaggart's vicious regress is nothing but a "benign" analysis of temporal attributions, he attempts to push his point even further by characterising higher-level moments as an unnecessary hypothesis. Were it so, evidently, no levels of predication would be available above the first one, and the infinite regress would be a fortiori untenable.

Following Smith's suggestion (1986, pp. 185-6), a standard analysis such as

(i) E is present at a moment which is present, and this moment is present at a second-level moment which is present, and this second-level moment is present at a third-level moment which is present, and so on, infinitely

could legitimately be reduced to

(ii) E is present at a moment which is present, and the being present of this moment is present, and the being present of the being present of this moment is present, and so on, infinitely 
and eventually to

(iii) $E$ is present, and the being present of $E$ is present, and the being present of the being present of $E$ is present, and so on, infinitely

by doing away with the moment-event distinction.

While I am quite sympathetic with assimilating events to their temporal location, I believe the first passage of Smith's reduction rests on a misunderstanding, which the geometric scheme helps bringing out. As I remarked while constructing the sequence of moments $\left\{m_{n}\right\}$, every further level of predication entails the addition of a new instant $m_{n+1}$ between 0 and $m_{n}$. In other words, the supposed "higher-level" moment is a second-level term in the logical ordering: far from resting on the pre-existing set of predicates, it is essential to them as a point of reference.

The slip is probably a direct consequence of the fact that the grounding element of the entire analysis (the subject's actual present) is unexpressed: this leaves event $\mathrm{E}$ as the only "solid" reference for the sequence, and may possibly induce to take it as a starting point for counting the levels of predication - thus reversing the correct logical order.

To complete the discussion about Smith's suggestion, it might be remarked that his simplified analysis entails either a contradiction or a tautology. In a sentence like (iii), the position of each moment is evaluated with respect to itself - which makes most decompositions impossible, since we would need to ascribe different coordinates to the same moment. The only exceptions are the three sequences where all $m_{i}$ 's overlap for any $i \geq 1$, that is, .. $00000, \ldots 0000-$ and ... $0000+-$ all corresponding to the truistic statement that a given moment coincides with itself? 


\section{ON SOME PARALLEL ARGUMENTS}

The mathematical interpretation of the A-series regress has an immediate consequence: if this part of McTaggart's paradox ultimately depends on geometric considerations, it seems reasonable to expect that the same argument be applicable in non-temporal contexts namely, for the study of space and for all those aspects of reality admitting of an analogy with space (just as it is the case with time itself).

In this section I will discuss at length a few significant examples of such non-temporal parallels; before getting into any detail, though, I need to introduce some basic temporal variants of McTaggart's regress.

\section{Temporal analogues}

Although McTaggart's argument is designed for a three-valued frame of temporal attributions, it can be easily restated for a two-valued system. The simplest way to do this is by equating any two of the three standard predicates.

Let us suppose, for example, that the present be assimilated to the future: in this case, the admissible attributions will be - and the couple of equivalent symbols $(\mathbf{0},+)$, which we may choose to represent with + . Applying McTaggart's analysis produces what I will call a two-valued oriented regress (as opposed to the original three-valued scheme). Both the complete diagram and the underlying summation rule can be obtained by substituting + for any occurrence of $\mathbf{0}$ and by eliminating the iterated strings of symbols, as is shown in pictures 4 and 5. It may be checked that the final results are consistent: that is, repeated applications of the modified summation rule generate precisely the modified diagram.

On the other hand, by regarding the present as part of the past, we will obtain the exact converse of the situation above. Since both the split-up rule and the diagram in the previous case are symmetric, they 
will obviously remain unchanged; incidentally, this common regressive scheme (see picture 5) is also the same that we would get by considering the present attribution as altogether impossible.

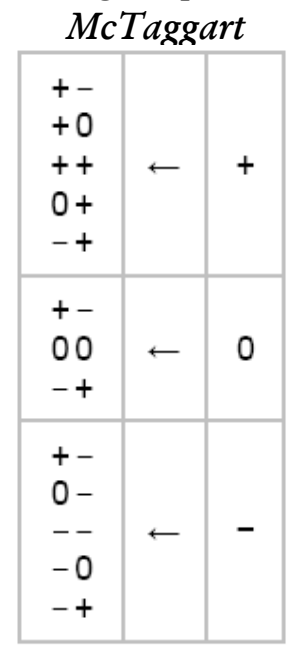

$$
\text { present }=\text { past }
$$

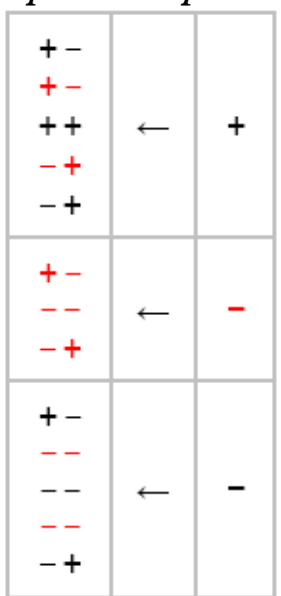

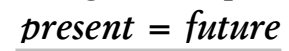
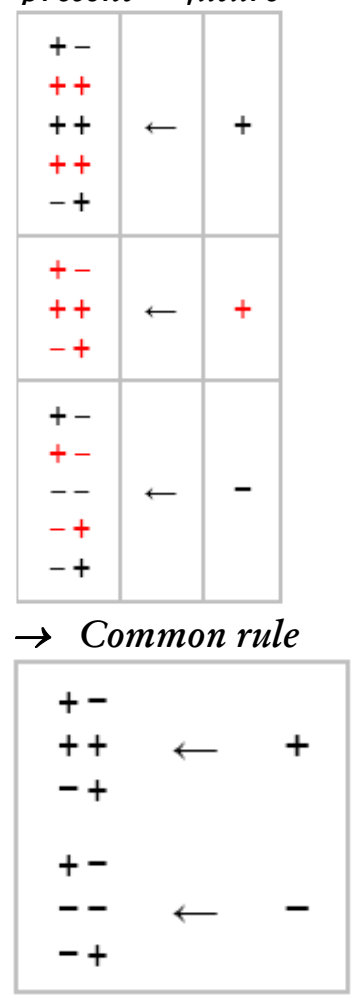

PICTURE 4. Split-up rules for bivalent oriented models. The unnecessary ombinations are highlighted in red: please notice that they exactly coincide with the ines including the symbol $\mathbf{0}$.

Manuscrito - Rev. Int. Fil., Campinas, v. 35, n. 2, p. 233-267, jul.-dez. 2012. 


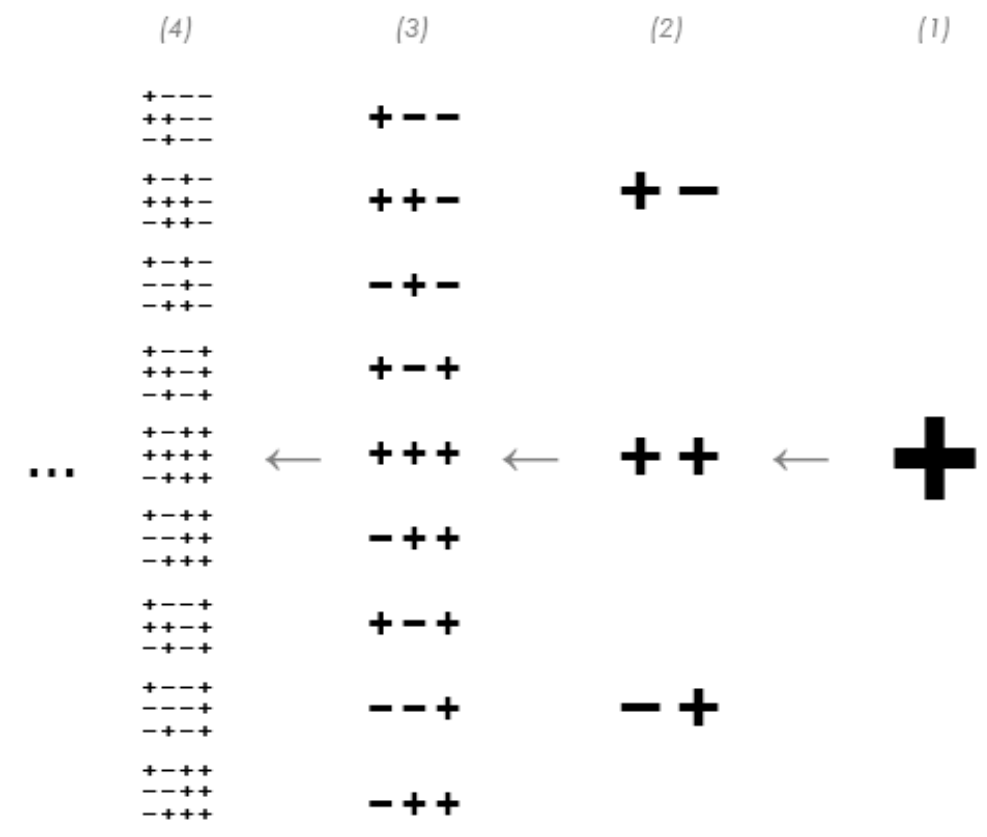

$\rightarrow$ Summation rule

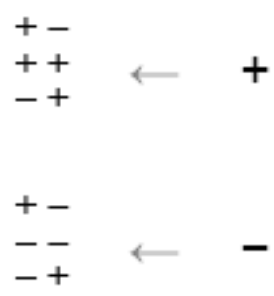

PiCture 5. Regress in a bivalent oriented scheme. The regress for the negative attribution may be obtained by reversing all the predicates. Both schemes hold independently of which sign we choose to attribute to 0 .

Manuscrito - Rev. Int. Fil., Campinas, v. 35, n.2, p. 233-267, jul.-dez. 2012. 
A much less natural possibility is to associate the past and the future. In this case, the scheme singles out the actual present but does not distinguish between any other two points on the timeline: we might call it a two-valued non oriented model.

Again, we can work out both the regress and the split-up rule in a mechanical way: in figure 6, I have chosen $\mathbf{X}$ as a single representative to replace the positive and negative symbols. Both schemes coincide with the intuitive results we would get by interpreting $\mathbf{0}$ and $\mathbf{X}$ respectively as 'equal' and 'different' (with respect to the actual present or any other point of reference). In this case, though, no nice algebraic interpretation is available.

\section{Modal analogues}

Since time is characteristically oriented, the binary regress shown in picture 6 is not very informative as a temporal scheme. On the other hand, the same diagram seems particularly well-suited for a general modal context, i.e. a possible world semantics whose basic structure may be reduced to the dichotomy of the actual and the merely possible $^{6}$. There is an obvious analogy between presentness and actuality in their respective frameworks, which means the regress described above can be applied as it stands to the modal case.

This particular non-temporal parallel has already been introduced in the literature on McTaggart. An article by M.J. Cresswell (1990) has a scheme comparable to mine - though he does not represents it on a diagram and offers no mathematical reading of how the regress is generated. Incidentally, Cresswell remarks that modality lacks a number of features which are peculiar to more specialized formal structures such as temporal ones: namely, the set of possible worlds has neither a

${ }^{6}$ As we will see, similar oppositions may be found at the root of other common indexicals.

Manuscrito - Rev. Int. Fil., Campinas, v. 35, n. 2, p. 233-267, jul.-dez. 2012. 
natural metric nor a complete ordering, or even a partial one for that matter. The well-known notion of similarity of worlds, introduced by Stalnaker (1968) and Lewis (1973), is too vague to be seriously considered as a general criterion of classification.

Yet, there is something in the standard modal semantics which might ground a basic structural asymmetry (with respect to the actual world) and thus somehow replace the natural direction of time in the A-series: it is the notion of relative accessibility of a world.

What could be the motive for analysing this concept with the tool of McTaggart's regress of attributions? Evidently, as in the temporal context, the need to single out objectively an element (here, a world) simply by using subjective properties - i.e., properties that are relative to a given world. In the case of modality, sometimes this procedure turns out to be successful: it is not difficult to imagine some examples of frames whose worlds make up an irregular network of accessibility relations; when this happens, it will be reasonable to expect that a McTaggart-style regress will be neither vicious nor infinite. For example, in any deontic frame, the actual world may be described by the same attribution (non-accessible) with respect to any possible world, including itself, which means contradiction never arises ${ }^{7}$. More generally, the final consistency of the predicate analysis will depend on the algebraic properties of accessibility - reflexivity, transitivity, and the like - and must therefore be assessed in each specific case.

${ }^{7}$ Furthermore, it is the unique world in the frame with such a property. Manuscrito - Rev. Int. Fil., Campinas, v. 35, n.2, p. 233-267, jul.-dez. 2012. 


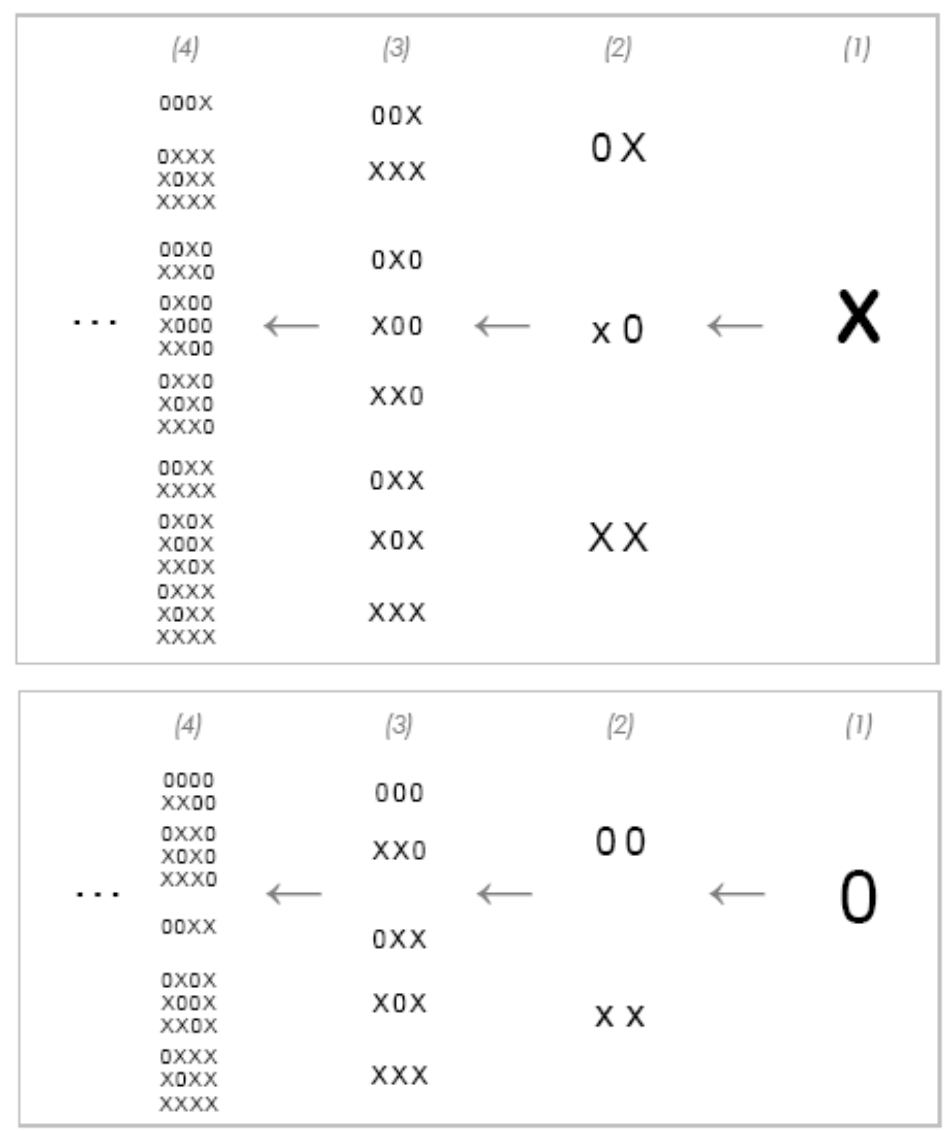

\section{$\rightarrow$ Split-up rule}

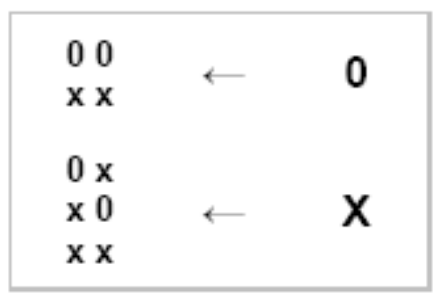

PiCture 6. Bivalent non-oriented regress for $\mathbf{0}$ and $\mathrm{X}$.Let us then restrict ourselves to a particular class of modal frames: is it possible to analyse temporal attributions in modal terms, via the notion of accessibility? 
Let us then restrict ourselves to a particular class of modal frames: is it possible to analyse temporal attributions in modal terms, via the notion of accessibility?

Before answering, we need to decide which kind of accessibility we want to consider. Every system of temporal logic involves at least two such relations, one for each direction: we can choose either one as our basic notion for defining tensed attributions, but not both at once, since part of the final goal is precisely to characterise the future with respect to the past. Moreover, it is necessary to assume a specific topology for time: for example, just to mention the main alternatives, linear (as in Mc'Taggart's argument) or branching.

These decisions are not arbitrary, but ultimately depend on some basic ontological presuppositions: what is it that we define as 'being real for a temporal entity? For example, by associating accessibility to the past, we would confer it a reality comparable with that of present events; conversely, if we only regard possible futures (single or multiple) as accessible, we are implying that what counts as real is what is obtaining now, or still has a chance to do so in the future ${ }^{8}-$ a past existence, at least when seen from the present, is literally nothing.

The first route has been chosen by John Bigelow (1991) ${ }^{9}$, who used precisely the notion of accessibility in order to characterise the past-present pair (as opposed to the future). Yet, the "worlds" he takes into account do not extend beyond the set of moments on a standard real timeline; as a result, his move turns out to be a pure linguistic device, and the reduction of pastness to accessibility boils down to a trivial case of identity ${ }^{10}$. In order to have a real definition of tenses in

${ }^{8} \mathrm{I}$ am taking for granted that events never happen twice; otherwise, what is susceptible of being repeated should equally be accepted as real.

9 For another modal analogue of McTaggart's paradox, see Buller and Foster (1992).

10 The reason why Bigelow (as well as Buller and Foster) gave such a limited interpretation of accessibility, is because none of them wanted to Manuscrito - Rev. Int. Fil., Campinas, v. 35, n.2, p. 233-267, jul.-dez. 2012. 
modal terms, it would be necessary to embed the A-series in the whole set of possible worlds, and evaluate accessibility in this larger space ${ }^{11}$ : for the sake of simplicity, we may identify the latter with the Cartesian plane, and the A-series itself with the $x$ axis.

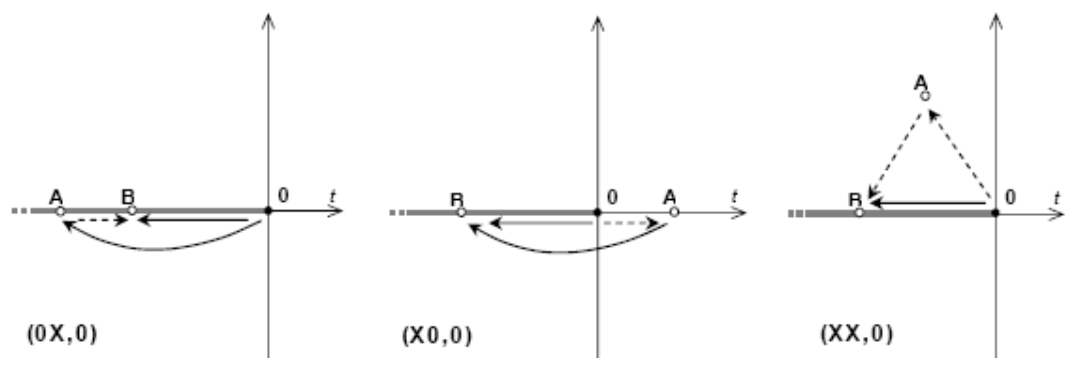

PiCTURE 7. Modal analysis of pastness in the linear case: three ways of breaking down the $\mathbf{O B}$ vector and the corresponding triplets. Continuous and dotted arrows stand respectively for accessibility and non-accessibility.

develop a parallel of McTaggart's proof. Their purpose was to use modal notions inside the original temporal regress, alternating them with tenses, in an attempt to construct a non-vicious analysis of the A-series attributions. The possibility of such a "hybrid" regress is a stimulating idea and deserves a specific discussion, but I will leave it aside since it is completely unrelated to the theme of this paper.

${ }^{11}$ Moreover, since the final purpose is an internal distinction between the two halves of the timeline, we would need a further partition between temporal non-accessible worlds (future instants) and purely modal ones; this could only be possible (in modal terms) by resorting to the notion of "future accessibility". Therefore, in a linear temporal framework, both "positive" and "negative" accessibility have to appear in the same analysis - regardless of which one I start it with.

Manuscrito - Rev. Int. Fil., Campinas, v. 35, n. 2, p. 233-267, jul.-dez. 2012. 
What will the split-up rule look like in this higher-dimensional framework? Just as tenses depend on the relative position of two instants (one of which is the actual present), accessibility is a function of a pair of points in the Cartesian plane ${ }^{12}$; the vector connecting two such points may be split in infinitely many ways as a sum of two other vectors, according to the parallelogram law, and therefore, the associated attribution may break down into any of the corresponding pair of values. If we call $u$ and $v$ the output variables, and $w$ the original attribution, we can represent the split-up rule through a set of admissible triplets $(u v, w)$, that is, all triplets corresponding to at least one combination of points in the plane. Since there exist only two values - accessibility and its converse: say, respectively, $\mathbf{0}$ and $\mathbf{X}$ - possible triplets are eight. In the case studied by Bigelow, it could be shown that all are admissible (see figure 7 for some examples) except $(\mathbf{0} 0, \mathbf{X})$, since the sum of two negative horizontal vectors is still negative and horizontal; by symmetry, the same holds in a linear model of time with future-oriented accessibility.

The resulting split-up rule is shown in picture 8: the set of pairs on the left-hand side are the same as in the non-oriented bivalent scheme of picture 6, with two additions. Consequently (by induction), each column of the regress of attributions will display more combinations that it did on that occasion, and since we already had contradiction at any stage, the same will be true a fortior in the present case.

${ }^{12}$ It is implied that accessibility may also be defined with respect to points outside the timeline: this is a necessary assumption if we want to allow for non-temporal intermediate points, and hence extend McTaggart's analysis. On the contrary, we do not have to assume that each vector is associated to a unique value (accessible-not accessible), invariant under translation: doing so would amount to resolving (quite unintuitively) the space of possible worlds into a sheaf of parallel chronologies, and it would make the case of branching time, which I will discuss later, virtually untreatable.

Manuscrito - Rev. Int. Fil., Campinas, v. 35, n.2, p. 233-267, jul.-dez. 2012. 


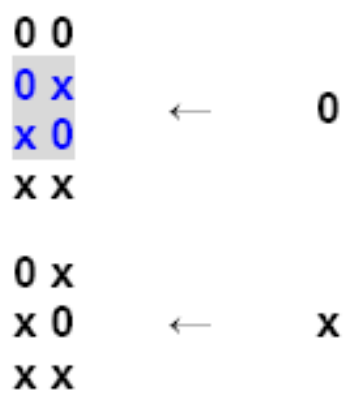

PICTURE 8. Split-up rule for the modal analysis of pastness. The highlighted pairs are those that were not present in picture 6 .

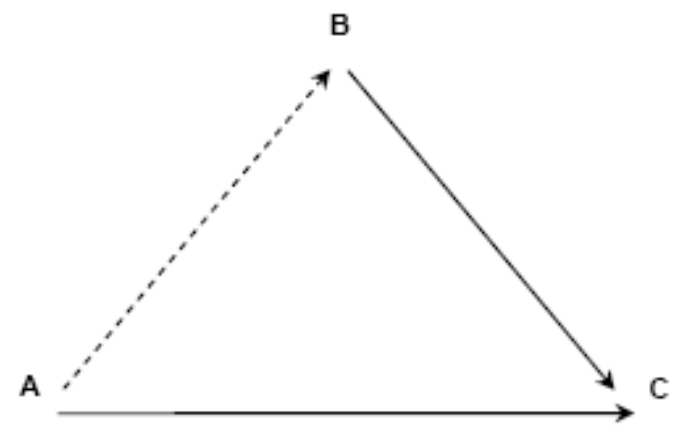

Picture 9. The disposition corresponding to the triplet $(\mathbf{X ~ 0 , 0 )}$. On the hypothesis that no arrow may point towards the past, a forward-branching model does not support such disposition.

Let us now turn to branching time. The common alternative here is between future-branching and double-branching models (a pastbranching structure is formally possible, but metaphysically of little interest). Either way, accessibility must be defined on the non-linear side, otherwise we would fall back into the previous hypothesis; as a result, a future-oriented accessibility will be admissible with both sorts of models, whereas a past-oriented one can only be defined in a double-branching case.

Manuscrito - Rev. Int. Fil., Campinas, v. 35, n. 2, p. 233-267, jul.-dez. 2012. 
Moreover, let us suppose for the moment that possible worlds reduce to those which are connected, directly or indirectly, to the actual present, without any further embedding.

Once again, the split-up rule can be checked through vector summation, by associating a characteristic diagram to each of the possible triplets: which of these diagrams will be compatible with a given branching model?

As in the linear case, the triplet $(\mathbf{0} \mathbf{0}, \mathbf{X})$ will have to be ruled out, since the accessibility relation is transitive in all the models we are considering. The remaining combinations will be acceptable with any kind of branching framework: each of them, as we saw, is exemplified by a set of instants on the real line, and since linear time can always be embedded in a branching model, as one of several alternative histories, the exemplification will be a valid one in such a model as well. Therefore, the set of possible triplets will be just the same as we found for a linear topology, and the regress will again be a vicious one ${ }^{13}$.

13 A further triplet, namely $(\mathbf{X ~ 0 , 0})$, may turn out to be inadmissible, provided we stipulate that moments in the past do not count as possible worlds (which means that no vector should point "backwards"). Under such a condition, $(\mathbf{X ~ 0 , 0})$ could not be instantiated in a forward-branching model, since it would imply that a single instant be future with respect to (and hence, accessible from) two distinct and unrelated moments, as shown in picture 9; on the other hand, a double-branching framework would allow for such a situation, and hence support the triplet.

In the hypothesis of forward-branching time, then, the split-up rule would only include six triplets - the same as in the scheme of picture 6 , plus $(\mathbf{0} \mathbf{X}, \mathbf{0})$ : since that regress was vicious, the same will hold in this case.

Manuscrito - Rev. Int. Fil., Campinas, v. 35, n.2, p. 233-267, jul.-dez. 2012. 


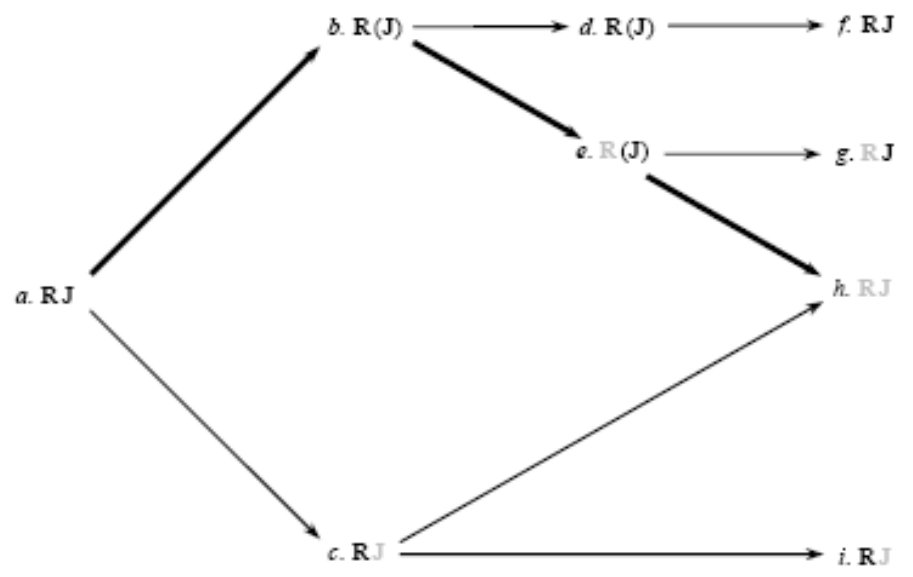

PiCture 10. A double-branching model. The scheme represents the conclusion of Romeo and Juliet, the thicker arrows correspond to the original sequence of events, while the others are alternative stories. A black letter stands for a character who is still alive; when it is grey, he/she is dead; the $\mathbf{J}$ in brackets means that Juliet is asleep after drinking the potion.

In order to illustrate part of these results, and to give some concreteness to the notion of accessible triplets, I have worked out the example in picture 10, which is inspired by Shakespeare's Romeo and Juliet. The model is branching on both sides, and has a path $(a, b, e, h)$ exemplifying the final sequence of events in the tragedy: Juliet drinks Friar Laurence's potion and falls asleep (b); Romeo believes her dead and swallows the poison (e); Juliet wakes up, finds him dead and stabs herself $(b)$. The other routes in the diagram represent some alternative courses of events: Romeo does not commit suicide and Juliet eventually wakes up $\left(b, d_{2} f\right)$; he does kill himself, but she doesn't $\left(e_{2} g\right)$; or else, she dies for real in the first place instead of taking the potion (the two lower paths).

From a formal point of view, accessibility is taken as futureoriented, in the sense that it holds for pairs where the second element is 


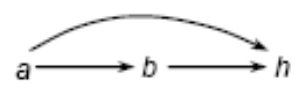

$(00,0): a, b, h$

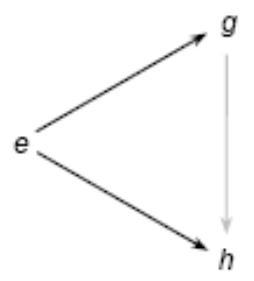

$(0 \mathrm{X}, 0): \mathrm{e}, \mathrm{h}, \mathrm{g}$

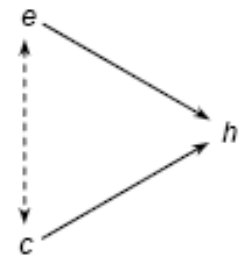

$(\mathbf{X} 0,0): e, c, h / c, e, h$

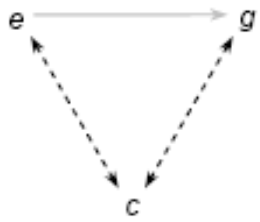

$(X X, 0): e, c, g$

$(0 \mathrm{X}, \mathrm{X}): e, g, \mathrm{C}$

$(\mathbf{X} 0, \mathrm{X}): c, e, g$

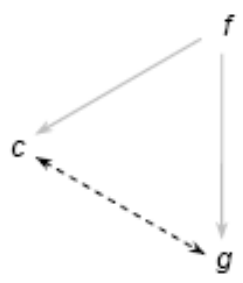

$(\mathbf{X X}, \mathbf{X}): c, g, f$

PiCture 11. Admissible decompositions in the double-branching case. The partial diagrams are taken from the model of picture 9, with letters indicating their exact position in the original net. A black continuous arrow represents succession within the same story, as above; a grey line stands for accessibility without temporal order, and the dotted double arrow means that the two terms are unaccessible to each other. Evidently, succession implies accessibility a fortiori.

The captions show the triplets and, for each of them, the logical order of the three knots.

still a possible outcome from the point of view of the first; in particular, future events in the same path will be trivially accessible. In the partial schemes (picture 11), knots are extracted by groups of three from the 
larger diagram, highlighting in each case which relations hold and which don't: it is easy to check that, on the whole, all the acceptable triplets are exemplified; furthermore, the result holds even if past moments do not count as possible worlds, since backward-oriented relations have not been employed in any of the examples.

Incidentally, it may be observed that the split-up rules for branching models remain unchanged if non-temporal possible worlds (i.e., points that are not connected to the present) are included in the framework.

The proof is quite straightforward. Whenever we add some extra knots to an existing diagram, all the previously admissible triplets will continue to hold, since each of them needs only to be instantiated by a single set of points. In addition, new relations might be established and new triplets be added to the list.

Yet, this is clearly not the case with our branching schemes. Suppose we extend the model to an extra set of points which are not temporally connected to the present, and suppose we take them into account (as possible intermediate points) when breaking down a path between two moments $\mathrm{A}$ and $\mathrm{B}$. Being disconnected from the temporal tree, all these external points will be inaccessible from both $\mathrm{A}$ and $\mathrm{B}$, and vice-versa; consequently, all the "new" triplets will be either $(\mathbf{X X}, \mathbf{0})$ or $(\mathbf{X X}, \mathbf{X})$. Since these combinations were already admissible, the non-temporal worlds add nothing to the split-up rule.

It is worth noticing that the modal analysis of tenses is partly dependent on whether the present is counted as accessible. So far, I have assumed that this is always the case, regardless of whether the relation is backward-oriented or forward-oriented. Incidentally, the acceptable triplets in each case would be exactly the same if presentness were not even considered as a possible attribution: after all, none of the examples I have illustrated in the pictures concerns vectors of zero length. 
On the other hand, let us suppose that the present tense is taken into account, but that accessibility is strictly associated either with the past or with the future: in other words, it is a non-reflexive relation. Let us now consider vector sums $\overrightarrow{\mathrm{AB}}+\overrightarrow{\mathrm{AC}}=\overrightarrow{\mathrm{BC}}$ such that two of the endpoints, or even all three, coincide: the corresponding triplets $(\mathbf{X X}, \mathbf{X}),(\mathbf{0} \mathbf{X}, \mathbf{0})$ and $(\mathbf{X ~ 0 , 0})$, will all turn out to be admissible. This adds nothing to the set of triplets we found previously, both for the linear and the branching case, except that $(\mathbf{X ~ 0 , 0})$ had been ruled out on special conditions for future-branching models (see note). From the point of view of the infinite regress, however, nothing changes: contradiction arises independently of whether the extra triplet is included or not in the split-up rule.

\section{Spatial analogues}

As the very structure of Mc'Taggart's regressive analysis is a spatial one, analogies in this field are far simpler and more numerous than their modal counterparts; for the sake of precision, one could even point out that all other applications (including the standard temporal version!) are extensions of the spatial case, and not conversely. The following is just a short review of the most intuitive cases of spatial regress.

A trivial possibility would be to adopt the temporal regress as it stands - either the complete three-valued model or any one of the binary versions. Quite evidently though, this would entail forcing space into the Procrustes' bed of an unlikely one-dimensional topology which is why this kind of option is not particularly interesting.

A much more natural choice is to adjust the geometric scheme to a higher-dimensional context (presumably a three-dimensional one). The logical construction should remain the same as in the temporal Cartesian line: an infinite sequence of points with one extreme on the actual 'here' and another coinciding with the location of a given object (or event), along with a corresponding sequence of attributions, each 
describing the position of a point with respect to the previous one. Clearly, the one-dimensional timeline should be replaced by an orthogonal frame whose origin would coincide with the actual 'here'.

The main problem with a higher-dimensional extension concerns the nature of spatial attributions. A simple qualitative distinction between two opposite directions, e.g. left and right, is no longer a viable option: although theoretically possible, it would not be nearly as

informative as the past-future dichotomy, and most important yet, it would require an arbitrary choice of a privileged spatial axis.

The most reasonable option seems to be that of a quantitative attribution, with full Cartesian coordinates describing the exact location of each point with respect to its antecedent in the sequence.

An interesting alternative is, once again, the non-oriented pair of attributions - in this context, 'here' and 'elsewhere'. This kind of solution is available for all types of demonstratives: besides the couples present/non-present and actual/non-actual, a further application could be imagined for the case of personal identity (I/non-I). The latter is perhaps the paradigm case of an indexical, as it directly expresses that opposition of an objective and a subjective perspective which is also mirrored in spatial, temporal, and modal attributions ${ }^{14}$. The binary scheme, "minimalist" as it is, allows us to capture such opposition; the failure of the predicate analysis reveals precisely the impossibility to account for the first-person perspective in objective terms.

14 Fine (2005, \12, pp. 310-8) deals extensively with personal identity, defending a kind of plural subjectivism which is the analogue of the temporal A-series, and even suggesting that the availability of such a view (provided one subscribes to his arguments) could serve as a guide for the standard McTaggart's paradox.

Manuscrito - Rev. Int. Fil., Campinas, v. 35, n. 2, p. 233-267, jul.-dez. 2012. 
(4)

(3)

$+-\boldsymbol{+} p$
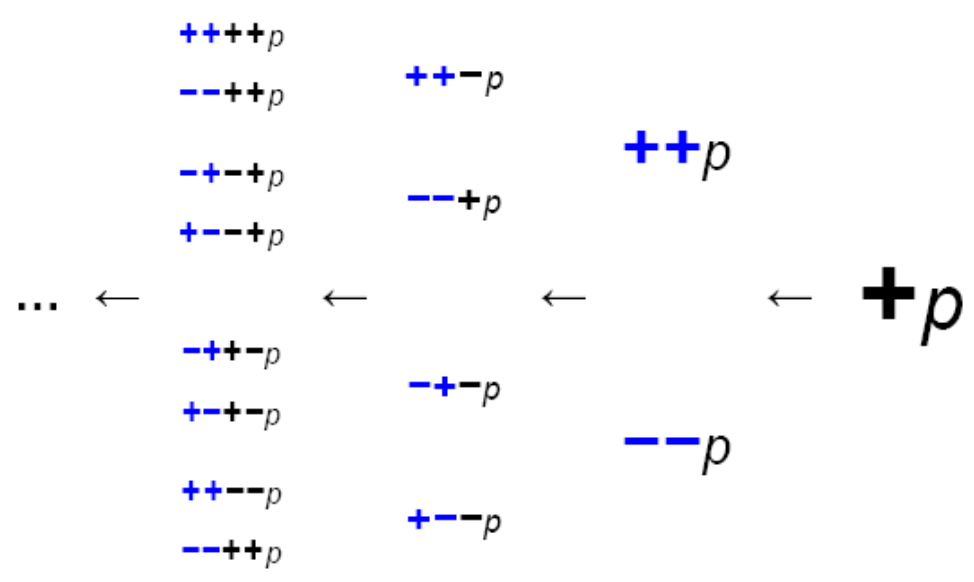

(2)

(1)

PICTURE 12. Rankin's infinite regress of truth values. The signs + and - stand respectively for truth and falsehood.

\section{A pure bivalent model: Rankin's paradox}

Despite their link with indexicality, the two-valued schemes studied so far seem external and to some extent superimposed on the reality they describe (whether it be temporal, spatial or modal), which they may be suspected to unduly simplify. In order to complete my review of parallel paradoxes, I will now introduce a fairly unorthodox example of a binary regressive pattern, defined on a discrete space consisting precisely of two points.

The "space" in question is the set of truth values in classical logic, which is also the pair of attributions appearing in the sequence. Just like in the previous examples, we may construct the infinite regress by taking either value as a starting point. The split-up rule, which coincides with columns 1 and 2 of picture 12, might be read more or less in these terms: 'It is true that $\mathrm{T}$ is true, it is false that $\mathrm{T}$ is false', or else ' $\mathrm{T}$ is true 
with respect to truth (the "T"' on the left), false with respect to falsity'. Needless to say, a similar interpretation holds for the analysis of $\mathrm{F}$.

Interestingly enough, this rule has a simple algebraic representation which is not the same as in the other cases: if the symbols + and - are taken in their standard value, we obtain the familiar sign rule for the product of real numbers ${ }^{15}$.

This unusual two-valued scheme has been proposed by Kenneth Rankin (1981), in an attempt to prove indirectly that McTaggart's paradox is not an acceptable argument, since it apparently leads to an illogical conclusion, namely, that all that is true is also false, and conversely (or, to put it in the author's own words, that reality is unreal).

Rankin's intuition is certainly brilliant, but the fact that he chose reality as an object for his paradox brings about some unexpected difficulties. The main one is that his spectacular conclusion is nothing short of a disavowal of the principle of contradiction. Although this is not immediately evident, McTaggart's paradox is logically dependent on this principle - it is because of non-contradiction that he declares the temporal attributions "mutually incompatible". Therefore: no incompatibility, no vicious regress of the A-series; no regress, no applications by analogy and hence no unreality of reality. By attempting to disprove McTaggart's conclusions, Rankin simultaneously undermines his own argument.

15 A less obvious interpretation could be given by associating $\mathrm{T}$ and $\mathrm{F}$ respectively to 0 and 1 in the set $Z_{2}$ of integers modulo 2 : although it is not as intuitive and reverses the conventional reading of $\mathrm{T}$ and $\mathrm{F}$, it preserves the consistency of the rule in the different cases of infinite regress.

Manuscrito - Rev. Int. Fil., Campinas, v. 35, n. 2, p. 233-267, jul.-dez. 2012. 


\section{CONCLUSION}

After so much formal work, a legitimate question remains: does the mathematical reading I propose have some relevant philosophical implications?

Perhaps it does. As I remarked, the key result in the first section of the paper is that the A-series contradiction is essentially a geometric phenomenon, which could be described as the impossibility of defining an absolute frame of reference through an infinite succession of relative coordinate systems.

Because of this geometric character, the paradox is obviously extendible to space itself, as well as to any aspect of reality where an analogy with space is applicable ${ }^{16}$ : the detailed (and yet incomplete) account I worked out for modality is an example of such potential extensions.

Does this imply that McTaggart's proof as a whole admits of nontemporal parallels? Not necessarily: it simply means that, if a genuinely temporal "ingredient" exists at all in the argument, it can only be found in the two preliminary steps.

Such a component can be easily identified with change, a key concept in both the first and the second implication of the proof. Broadly speaking, change may be described as the succession of two different states of affairs in two separate moments: in other words, it is a function of time, and consequently it has no consistent meaning outside a temporal perspective.

Unfortunately for McTaggart, there is a serious logical weakness in the way this notion is exploited in the proof: to put it very simply, 'change' in step 1 has not quite the same meaning as in step 2.

${ }^{16}$ It would be interesting to discuss whether a logical criterion exists for establishing the legitimacy of space analogies; personally, I am inclined to think that the acceptance of a given analogy depends essentially on an arbitrary decision.

Manuscrito - Rev. Int. Fil., Campinas, v. 35, n.2, p. 233-267, jul.-dez. 2012. 
The assertion that change involves the A-series is justified by McTaggart with the following argument: any change is an event; but what temporal qualities of this event may be said to change ${ }^{17}$ ? Certainly not the B-series relations of earlier and later, which hold independently of time; therefore, it must be the position with respect to the A-series the fact of being past, present or future.

On the other hand, in the first implication (time entails change), the underlying idea is that there is no such thing as a "pure" flow of time: duration, however small, is always associated to a variation in some real properties of things (mental or physical).

Quite clearly, though, this is not the kind of change he appeals to in the second step: in fact, the difference in the A-series positions represents precisely the pure temporal passage he explicitly rejects. Therefore the notion of change, as something distinct from time, has no concrete role in the economy of the proof.

If the notion of change, as it is exploited by McTaggart, does indeed give rise to a paralogism, the consequence is that the whole paradox should be rebuilt on new bases, with no intermediate notions between time and the A-series.

This constructive part, however, is well beyond the scope of the present paper. What had to be established, here, is that the argument constructed by McTaggart is neither a deceptive dialectical trick, nor an easy shortcut for a complex metaphysical issue. The paradox reveals something important on the nature of time, namely, that the way we intuitively conceive it - the A-series - harbours a contradiction; furthermore, this logical shortcoming is not peculiar to time, but concerns all forms of subjective experience of reality. Whether it is possible to rationally account for such experience, and in what forms, is

${ }^{17}$ Critics of McTaggart who hold a tenseless view, i.e. who take the B-series to be constitutive of time, typically reject this argument by arguing that features of events need not change at all: see for example Mellor (1981, p. 103).

Manuscrito - Rev. Int. Fil., Campinas, v. 35, n. 2, p. 233-267, jul.-dez. 2012. 
a question that cannot be answered from within the reassuring conceptual architecture of McTaggart's proof: in order to address it, we would have to step back from the mathematical structure and attempt an interpretation of it, which will presumably depend on fundamental (and non-negotiable) metaphysical presuppositions on reality, subjectivity, and the relation of the self with the world. McTaggart himself (most notably in The nature of existence) developed such a positive theory, which could be described as an ontology of communicating subjects; yet, the answer might as well take a different direction. The logical structure of the paradox does not put any constraint on the pars construens that will follow it: which is probably why, after a hundred years, it is still a vital source of philosophical reflection.

\section{REFERENCES}

BIGELOW, J. “Worlds enough for time”. Noûs, 25, pp. 1-19, 1991.

BULLER, D. J., FOSTER, T. R. “The new paradox of temporal transience”. Philosophical Quarterly, 168, pp. 357-366, 1992.

CRESSWELL, M.J. “Modality and Mellor's McTaggart”. Studia Logica, 49, pp. 163-170, 1990.

FINE, K. Modality and tense: philosophical papers. Oxford: Clarendon Press, 2005.

FINE, K. “Tense and reality”. In: Fine (2005), pp. 261-320.

LEWIS, D. Counterfactuals. Oxford: Blackwell; Cambridge, MA: Harvard University Press, 1973. 
MANCUSO, D. "Dai futuri contingenti all'irrealtà del tempo". Isonomia, sezione epistemologica, 2004. Available at

http://www.uniurb.it/Filosofia/isonomia/2004mancuso.pdf.

MCTAGGART, J. E. “The unreality of time”. Mind, 18, pp. 457-474, 1908. Revised and reprinted as "Time" in MCTAGGART (1927).

. The nature of existence, vol. II. Cambridge: Cambridge University Press, 1927.

MELLOR, D.H. Real time. Cambridge: Cambridge University Press, 1981.

PRIOR, A.N. Past, present, and future. Oxford: Clarendon Press, 1967.

RANKIN, K. “McTaggart's paradox: two parodies”. Philosophy, 56, pp. 333-348, 1981.

REICHENBACH, H. Elements of symbolic logic. New York: The Free Press; London: Collier-Macmillan, 1966. Originally printed in 1947.

RESCHER, N. (ed.). Studies in logical theory. Oxford: Blackwell, 1968.

SMITH, Q. “The infinite regress of temporal attributions”. Southern Journal of Philosophy, 24, 1986. Repr. in Smith and Oaklander (1995), pp. 180-194.

-., OAKLANDER, N. (eds.). The new theory of time. New Haven: Yale University Press, 1995.

STALNAKER, R. “A theory of conditionals”. In: Rescher (ed.) (1968), pp. 98-112.

Manuscrito - Rev. Int. Fil., Campinas, v. 35, n. 2, p. 233-267, jul.-dez. 2012. 